\title{
ON $S$-RINGS IN THE SENSE OF F. KASCH
}

\author{
KIITI MORITA
}

\author{
To the memory of TADASI NAKAYAMA
}

The notion of $S$-rings was introduced by F. Kasch [4] in establishing a theory of Frobenius extensions. $S$-rings possess several remarkable properties, although they have been shown to be not indispensable to the theory of Frobenius extensions. The purpose of this paper is to give some characteristic properties of $S$-rings.

1. Statement of the results. Throughout this paper let $A$ be an associative ring which has an identity element 1 and satisfies the minimum condition for left and right ideals. All modules are assumed to be unitary.

In case for any right ideal $J$ of $A$ with $J \neq A$ we have

$$
l(J) \neq 0,
$$

we shall say that $A$ is a left $S$-ring. Here we denote by $l(J)$ the left annihilator of $J$, that is,

$$
l(J)=\{a \mid a J=0, a \in A\} .
$$

Similarly we define right $S$-rings. $A$ ring which is a right $S$-ring as well as a left $S$-ring is nothing but an $S$-ring in the original sense of Kasch. As is seen from (II) of Theorem 1 below, $A$ is always an $S$-ring if $A$ is commutative.

Let $U$ be a left $A$-module and $V$ a right $A$-module. $A$ mapping $\omega: U \times V$ $\rightarrow A$ is called an $A$-bilinear form on $U \times V$ if $\omega$ is additive with respect to $u \in U$ and $v \in V$, i.e.

$$
\begin{aligned}
& \omega\left(u+u^{\prime}, v\right)=\omega(u, v)+\omega\left(u^{\prime}, v\right), \\
& \omega\left(u, v+v^{\prime}\right)=\omega(u, v)+\omega\left(u, v^{\prime}\right),
\end{aligned}
$$

and if

$$
\begin{aligned}
& \omega(a u, v)=a \omega(u, v), \\
& \omega(u, v a)=\omega(u, v) a, \quad \text { for } a \in A, u \in U, v \in V .
\end{aligned}
$$

Received July 1, 1965. 
An $A$-bilinear form $\omega$ on ${ }_{A} U \times V_{A}$ is said to be regular if

$$
\begin{aligned}
& \omega(u, v)=0 \text { for all } v \in V \text { implies } u=0, \\
& \omega(u, v)=0 \text { for all } u \in U \text { implies } v=0 .
\end{aligned}
$$

A left $A$-module $M$ is called a generator if there is a positive integer $n$ such that a direct sum of $n$ copies of $M$ has a direct summand which is $A$-isomorphic to ${ }_{A} A ; M$ is a generator if and only if there are a finite number of elements $m_{i} \in M, \varphi_{i} \in \operatorname{Hom}_{A}\left(M,{ }_{A} A\right), i=1, \ldots, n$, such that $\sum_{i=1}^{n} \varphi_{i}\left(m_{i}\right)=1$.

Now, with these definitions, our main theorem is stated as follows.

THEOREM 1. The following statements are equivalent.

(I) $A$ is a left $S$-ring.

(II) For any simple right $A$-module $M$ there is an $A$-submodule of $A_{A}$ which is $A$-isomorphic to $M$.

(III) For any finitely generated, non-zero right $A$-module $M$ we have $\mathrm{Hom}_{A}$ $\left(M, A_{A}\right) \neq 0$.

(IV) If $U$ is a finitely generated, projective left A-module and $V$ a right $A$ module, and if there is a regular A-bilinear form $\omega$ on $U \times V$, then we have an A-isomorphism: $V \cong\left[\operatorname{Hom}_{A}\left(U,{ }_{A} A\right)\right]_{A}$.

(IV)' If $U$ is a finitely generated, free left $A$-module and $V$ a right A-module, and if there is a regular A-bilinear form on $U \times V$, then we have an $A$-isomorphism: $V \cong\left[\operatorname{Hom}_{A}\left(U,{ }_{A} A\right)\right]_{A}$.

(V) If $U$ is a projective left $A$-module and $U^{\prime}$ is a finitely generated, projective, left $A$-submodule of $U$, then $U^{\prime}$ is a direct summand of $U$.

(V)' If $U$ is a free left $A$-module and $U^{\prime}$ is a finitely generated free $A$-submodule of $U$, then $U^{\prime}$ is a direct summand of $U$.

(VI) A projective left $A$-module $L$ is a generator if $L$ is faithful.

In case $A$ is an $S$-ring, statements (IV)' and (V)' are proved by Kasch [4], and (IV) and (V) (with $U$ finitely generated) by Nakayama-Tsuzuku [7]. The equivalence $(\mathrm{I}) \Longleftrightarrow(\mathrm{V})$ is proved by Bass [1] (cf. also Jans [3]). Our proof of Theorem 1 does not presuppose any of these results.

The property of $S$-rings which is stated as (VI) seems to have not been noticed in the literature. It is to be noted that $A$ is a quasi-Frobenius ring if and only if every faithful left $A$-module is a generator; this is seen from $[6$, Theorems 14.1 and 16.5]. 
The proof of the implication $(\mathrm{V})^{\prime} \Rightarrow(\mathrm{VI})$ is based on the following theorem which may be of interest by itself.

Theorem 2. A left A-module $M$ is faithful if and only if there is a positive integer $n$ such that a direct sum of $n$ copies of $M$ has an $A$-submodule which is $A$-isomorphic to ${ }_{A} A$.

As an application of Theorem 1 we obtain the following

Theorem 3. Let $A$ be a left S-ring and let $M$ be a right $A$-module which is a generator. Then the A-endomorphism ring $C$ of $M$ is a left $S$-ring if and only if $M$ is finitely generated and projective; if this is the case $C$ is similar to $A$.

Theorem 4. Every residue class ring of $A$ is a left $S$-ring if and only if $A$ is decomposed into a direct sum of two-sided ideals each of which is a primary ring.

TheOREM 5. $A$ is a quasi-Frobenius ring if and only if $A$ is a left S-ring and a $Q F-3$ ring.

In view of Theorem 5 it would be interesting to compare Theorem 4 with results of Kawada [5] and Ikeda [2].

2. Proof of Theorem 2. Since the "if" part is evident, we have only to prove the "only if" part. For this purpose, suppose that $M$ is faithful. Let us set

$$
J=\cap\left\{\text { Kernel } f \mid f \in \operatorname{Hom}_{A}\left({ }_{A} A, M\right)\right\} .
$$

Then $J$ is a left ideal of $A$. Let $a_{0}$ be any non-zero element of $A$. Then there exists an element $m_{0}$ of $M$ such that $a_{0} m_{0} \neq 0$. If we set $f(a)=a m_{0}$ for $a \in A$, then $f \in \operatorname{Hom}_{A}\left({ }_{A} A, M\right)$ and $a_{0} \notin$ Kernel $f$. Hence we have $a_{0} \notin J$. This shows that $J=0$. Since $A$ satisfies the minimum condition for left ideals, there exist a finite number of elements $f_{i}, i=1, \ldots, n$, of $\operatorname{Hom}_{A}\left({ }_{A} A, M\right)$ such that

$$
\bigcap_{i=1}^{n} \text { Kernel } f_{i}=0 \text {. }
$$

Now, let us set

$$
\varphi(a)=\left(f_{1}(a), \ldots, f_{n}(a)\right) \quad \text { for } a \in A ;
$$

then $\varphi$ is an $A$-homomorphism from ${ }_{A} A$ to $M^{(n)}$, where $M^{(n)}$ is a direct sum 
of $n$ copies of $M$. Since Kernel $\varphi=\bigcap_{i=1}^{n}$ Kernel $f_{i}, \varphi$ is a monomorphism by (10). Thus the "only if" part of the theorem is proved.

3. Proof of Theorem 1. If $J$ is a right ideal of $A$, then $l(J)=\operatorname{Hom}_{A}(A / J$, $A_{A}$ ). Hence the equivalence (I) $\Longleftrightarrow$ (III) is obvious. The equivalence (II) $\Leftrightarrow$ (III) is clear.

(III) $\Longrightarrow$ (IV). Assume (III). Let $U$ be a finitely generated, projective, left $A$-module and $V$ a right $A$-module. Suppose that $\omega$ is a regular $A$-bilinear form on $U \times V$. Then we set

$$
\begin{gathered}
V^{*}=\left[\operatorname{Hom}_{A}\left(U,{ }_{A} A\right)\right]_{A}, \\
\omega^{*}(u, v)=v(u), \quad \text { for } u \in U, v \in V^{*} .
\end{gathered}
$$

If we set $\varphi_{v}(u)=\omega(u, v)$ for $u \in U, v \in V$, then we have $\varphi_{v} \in V^{*}$ and $\omega^{*}\left(u, \varphi_{v}\right)$ $=\omega(u, v)$. Since $\varphi_{v}=0$ if and only if $v=0$, we can consider $V$ as an $A$-submodule of $V^{*}$. Assume that $V^{*} \neq V$. Then by (III) there is $g \in \operatorname{Hom}_{A}\left(V^{*} / V\right.$, $A_{A}$ ) such that $g \neq 0$. If we denote by $f$ the canonical projection from $V^{*}$ onto $V^{*} / V$, then we have $\psi=g \circ f \in \operatorname{Hom}_{A}\left(V^{*}, A_{A}\right)$. Hence there exists an element $u_{0}$ of $U$ such that

$$
\omega^{*}\left(u_{0}, v\right)=\psi(v) \quad \text { for } v \in V^{*} .
$$

By definition of $\psi$ we have $\psi(v)=0$ for $v \in V$. This shows that $\omega\left(u_{0}, v\right)=0$ for all $v \in V$. By the regularity of $\omega$, we have $u_{0}=0$, which contradicts the fact that $\psi \neq 0$. Thus we have $V^{*}=V$.

$(\mathrm{IV}) \Longrightarrow(\mathrm{V})$. Assume (IV). Let $U$ be a projective left $A$-module and $U^{\prime}$ an $A$-submodule of $U$ which is finitely generated and projective. Then there is a free left $A$-module $U_{0}$ such that $U$ is a direct summand of $U_{0}$. Since $U^{\prime}$ is finitely generated, there is a direct summand $U_{1}$ of $U_{0}$ which is finitely generated and contains $U^{\prime}$. If $U^{\prime}$ is a direct summand of $U_{1}$, then $U^{\prime}$ is a direct summand of $U_{0}$ and consequently $U^{\prime}$ is a direct summand of $U$. Therefore we may, and shall, assume without loss of generality that $U$ is finitely generated.

Let us set

$$
\begin{gathered}
V=\left[\operatorname{Hom}_{A}\left(U,{ }_{A} A\right)\right]_{A}, \\
\omega(u, v)=v(u) \quad \text { for } u \in U, v \in V, \\
R_{\omega}\left(U^{\prime}\right)=\left\{v \mid \omega\left(u^{\prime}, v\right)=0 \text { for all } u^{\prime} \in U^{\prime}\right\} .
\end{gathered}
$$


Then if $v-v^{\prime} \in R_{w}\left(U^{\prime}\right)$, we have $\omega\left(u^{\prime}, v\right)=\omega\left(u^{\prime}, v^{\prime}\right)$ for any $u^{\prime} \in U^{\prime}$. Hence if we set

$$
\omega^{\prime}\left(u^{\prime},\{v\}\right)=\omega\left(u^{\prime}, v\right) \quad \text { for } u^{\prime} \in U^{\prime},\{v\} \in V / R_{w}\left(U^{\prime}\right),
$$

$\omega^{\prime}$ is an $A$-bilinear form on $U^{\prime} \times V / R_{w}\left(U^{\prime}\right)$. Moreover $\omega^{\prime}$ is regular as is easily seen. Hence by (IV) we see that

$$
V / R_{\mathfrak{w}}\left(U^{\prime}\right) \cong \operatorname{Hom}_{A}\left(U^{\prime},{ }_{A} A\right) .
$$

Therefore $V / R_{w}\left(U^{\prime}\right)$ is projective and hence $R_{w}\left(U^{\prime}\right)$ is a direct summand. If we set

$$
L_{\omega}\left(V^{\prime}\right)=\left\{u \mid \omega\left(u, v^{\prime}\right)=0 \text { for all } v^{\prime} \in V^{\prime}\right\},
$$

then we have $L_{w}\left(R_{w}\left(U^{\prime}\right)\right) \supseteqq U^{\prime}$ and

$$
L_{\mathrm{w}}\left(R_{\mathrm{w}}\left(U^{\prime}\right)\right) \cong \operatorname{Hom}_{d}\left(V / R_{\mathrm{w}}\left(U^{\prime}\right), A_{A}\right) .
$$

Since $U^{\prime} \cong \operatorname{Hom}_{d}\left(V / R_{w}\left(U^{\prime}\right), A_{A}\right)$ by (16), this shows that $U^{\prime}=L_{w}\left(R_{w}\left(U^{\prime}\right)\right)$.

On the other hand, since $R_{w}\left(U^{\prime}\right)$ is a direct summand of $V$, we have

$$
\operatorname{Hom}_{A}\left(R_{w}\left(U^{\prime}\right), A_{A}\right) \cong U / L_{\omega}\left(R_{\omega}\left(U^{\prime}\right)\right)=U / U^{\prime} .
$$

Since $V$ is finitely generated and projective, so is also $R_{w}\left(U^{\prime}\right)$. Hence $U / U^{\prime}$ is projective. Therefore $U^{\prime}$ is a direct summand of $U$.

$(\mathrm{III}) \Rightarrow(\mathrm{IV})^{\prime},(\mathrm{IV})^{\prime} \Longrightarrow(\mathrm{V})^{\prime}$. These implications are readily seen from the proofs given above for the implications (III) $\Rightarrow($ IV), (IV) $\Longrightarrow(\mathrm{V})$.

$(\mathrm{V}) \Longrightarrow(\mathrm{V})^{\prime}$ is obvious.

$(\mathrm{V})^{\prime} \Longrightarrow(\mathrm{VI})$. Let $L$ be a projective, faithful, left $A$-module. Then by Theorem 2 there is a positive integer $n$ such that a direct sum $L^{(n)}$ of $n$ copies of $L$ has an $A$-submodule $L_{0}$ which is $A$-isomorphic to ${ }_{A} A$. Since $L^{(n)}$ is projective, there is a free left $A$-module $U$ such that $L^{(n)}$ is a direct summand of $U$. Now, assume $(\mathrm{V})^{\prime}$. Then $L_{0}$ is a direct summand of $U$. Since $L_{0} \subseteq L^{(n)}, L_{0}$ is also a direct summand of $L^{(n)}$. This shows that $L$ is a generator.

4. Proof of Theorem 1 (continued). Let ${ }_{A} \mathfrak{M}$ (resp. $\mathfrak{M}_{A}$ ) be the category of left (resp. right) $A$-modules. Let $B$ be another ring. If $S:{ }_{A} \mathfrak{M} \rightarrow{ }_{B} \mathfrak{M}$ and $T$ : ${ }_{B} \mathfrak{M} \rightarrow{ }_{A} \mathfrak{M}$ are covariant additive functors such that $S T$ and $T S$ are naturally equivalent to the identity functor, each of $S$ and $T$ is called a category-isomorphism. In case there is a category-isomorphism from ${ }_{A} \mathfrak{M}$ to ${ }_{B} \mathfrak{M}, A$ is said 
to be similar to $B$; $A$ is similar to $B$ if and only if there is a category-isomorphism from $\mathfrak{M}_{A}$ to $\mathfrak{M}_{B}$. Let $S:{ }_{A} \mathfrak{M} \rightarrow{ }_{B} \mathfrak{M}$ be a category-isomorphism. If $X \in{ }_{A} \mathbb{M}$ has one of the following properties:

(a) simple, (b) finitely generated, (c) projective, (d) faithful, (e) generator, then $S(X)$ has also the same property. Since $S$ is an exact functor, if $X^{\prime}$ is an $A$-submodule of $X$ (where $X, X^{\prime} \in{ }_{A} \mathbb{M}$ ) then $S\left(X^{\prime}\right)$ is $B$-isomorphic to a $B$ submodule of $S(X)^{1)}$.

Therefore we have the following lemmas.

Lemma 1. If $A$ is similar to $B$ and $A$ is a left $S$-ring, then $B$ is a left $S$ ring.

Proof. We have only to use the equivalence (I) $\Leftrightarrow$ (II) in Theorem 1 .

Lemma 2. If statement (VI) of Theorem 1 holds for $A$ and if $A$ is similar to $B$, then statement (VI) with $A$ replaced by $B$ holds.

Now, we shall prove the implication (VI) $\Longrightarrow(\mathrm{I})$. Since $A$ is similar to its basic ring (cf. [6]), by Lemmas 1 and 2 we may, and shall, assume without loss of generality that $A$ is a basic ring of itself.

Let $e_{1}, \ldots, e_{m}$ be mutually orthogonal primitive idempotents of $A$ such that $e_{1}+\cdots+e_{m}=1$. Then $A e_{i}$ is not $A$-isomorphic to $A e_{j}$ for $i \neq j$. Let us set

$$
L_{i}=\sum_{j \neq i} \oplus A e_{j}
$$

Then $L_{i}$ is finitely generated and projective, but it is not a generator. Now, assume (VI). Then $L_{i}$ is not faithful. Hence if we set

$$
J=\left\{a \mid a L_{i}=0, a \in A\right\},
$$

we have $J \neq 0$. Let $N$ be the radical of $A$. If $J N=0$, we set $J_{0}=J$. If $J N \neq 0$, there is a positive integer $i$ such that $J N^{i} \neq 0$ but $J N^{i+1}=0$; in this case we set $J_{0}=J N^{i}$. Then, in any case we have

$$
J_{0} \neq 0, J_{0}\left(e_{i} N\right)=0, J_{0}\left(\sum_{j \neq i} \oplus e_{j} A\right)=0 .
$$

Therefore we have

1) For the prcofs of these results cf. Morita [6]. 


$$
l\left(M_{i}\right) \neq 0, \text { for } M_{i}=e_{i} N \oplus \sum_{j \neq i} \oplus e_{j} A .
$$

Since $\left\{M_{1}, \ldots, M_{m}\right\}$ is the totality of maximal right ideals of $A$, we see that $A$ is a left $S$-ring.

Thus the proof of Theorem 1 is completed.

5. Proof of Theorem 3. If a right $A$-module $M$ is a finitely generated, projective generator, the functor $P:{ }_{A} \mathfrak{M} \rightarrow{ }_{C} \mathfrak{M}$ defined by $P(X)={ }_{C} M_{A} \otimes{ }_{A} X$ is a category-isomorphism where $C=\operatorname{End}_{A}\left(M_{A}\right)$ since in this case the bimodule,$M_{A}$ satisfies the conditions of [6, Theorem 3.4] by [6, Lemma 3.3]. Hence the "if" part of Theorem 3 follows readily from Lemma 1 .

To prove the "only if" part, suppose that $C$ is a left $S$-ring. Then ${ }_{c} M$ is faithful. Since $M_{A}$ is a generator, ${ }_{c} M$ is finitely generated and projective by [6, Lemma 3.3]. Hence by Theorem $1_{c} M$ is a generator. Since $A$ is inverseisomorphic to the $C$-endomorphism ring of $c M$ by [6, Lemma 3.3], we see by [6, Lemma 3.3] again that $M_{A}$ is finitely generated and projective. Thus the "only if" part is proved.

6. Proof of Theorem 4. It suffices to prove the "only if" part. For this purpose, suppose that every residue class ring of $A$ is a left $S$-ring. Let $e_{i \kappa}$, $\kappa=1, \ldots, f(i), i=1, \ldots, n$, be mutually orthogonal primitive idempotents of $A$ such that $A e_{i \kappa} \cong A e_{j \lambda}$ if and only if $i=j$, and that $\sum_{i=1}^{n} \sum_{\kappa=1}^{f(i)} e_{i \kappa}=1$. Let us set $e_{i}=e_{i 1}$ and

$$
z_{i}=l\left(A e_{i}\right), \quad i=1, \ldots, n .
$$

Then it is easily verified that $A e_{i}$ is faithful, projective, and indecomposable as a left $A / z_{i}$-module. Hence, by the assumption and by Theorem $1, A e_{i}$ is a generator as a left $A / z_{i}$-module. Therefore $A / z_{i}$ is a primary ring.

Let $N$ be the radical of $A$. Then $\widetilde{N}=\left(N+z_{i}\right) / z_{i}$ is the radical of $\widetilde{A}=A / z_{i}$. Let us denote by $\tilde{e}_{j \lambda}$ the residue class of $e_{j \lambda}$ modulo $z_{i}$. If $\widetilde{A} \widetilde{e}_{j \lambda} / \widetilde{N} \widetilde{e}_{j \lambda} \neq 0$, then $\left(N+\gamma_{i}\right) e_{j \lambda}=N e_{j \lambda}$ and $\widetilde{A} \widetilde{e}_{j \lambda} / \widetilde{N} \widetilde{e}_{j \lambda} \cong A e_{j \lambda} / N e_{j \lambda}$ as left $\widetilde{A}$-modules, while $\widetilde{A} \widetilde{e}_{j \lambda} / \tilde{N} \widetilde{e}_{j \lambda}$ $=0$ implies $e_{j \lambda} \in z_{i}$. Since $\widetilde{A}$ is primary as is proved above and $e_{i} \neq 0$, we have $e_{j \lambda} \in \delta_{i}$ for $j \neq i$. This shows that

$$
\sum_{j \neq i} \sum_{\lambda=1}^{(j)} \oplus A e_{j \lambda} \leqq \delta_{i} .
$$


On the other hand, if $a \in z_{i}$, then $a e_{i \kappa}=0$ for any $\kappa$ since $z_{i}=l\left(A e_{i \kappa}\right)$, and hence $a \in \sum_{j \neq \imath} \sum_{\lambda} \oplus A e_{j \lambda}$.

Thus we have

$$
z_{i}=\sum_{j \neq i} \sum_{\lambda=1}^{f(\jmath)} \oplus A e_{j \lambda}
$$

Hence, if we set

$$
\mathfrak{w}_{i}=\sum_{\kappa=1}^{f(i)} \oplus A e_{i \kappa},
$$

then

$$
\mathfrak{w}_{i}=\bigcap_{j \neq i} z_{j}
$$

Thus $\mathfrak{w}_{i}$ are two-sided ideals of $A$ and

$$
A=\sum_{i=1}^{n} \oplus \mathfrak{w}_{i}
$$

Since $\mathfrak{w}_{i}$ is ring-isomorphic to $A / z_{i}, \mathfrak{w}_{i}$ is a primary ring. Therefore the "only if" part is proved.

7. Proof of Theorem 5. In case $A$ has a unique minimal faithful left $A$-module, $A$ is called a $Q F-3$ ring; this is the case if and only if there is a faithful, projective, injective left A-module (cf. [5]) (e.g., this follows readily from Theorem 2). Hence, if $A$ is a $Q F-3$ ring and a left $S$-ring, we see by Theorem 1 that $A$ is quasi-Frobenius. Since the "only if" part is obvious, this proves Theorem 5 .

\section{REFERENCES}

[1] H. Bass, Finitistic dimension and a homological generalization of semi-primary rings, Trans. Amer. Math. Soc., 95 (1960), 466-488.

[2] M. Ikeda, Some generalizations of quasi-Frobenius rings, Osaka Math. J., 3 (1951), 227-238.

[ 3 ] J. P. Jans, Duality in Noetherian rings, Proc. Amer. Math. Soc., 12 (1961), 829-835.

[4] F. Kasch, Grundlagen einer Theorie der Frobeniuserweiterungen, Math. Ann., 127 (1954), 453-474.

[5] Yutaka Kawada, A generalization of Morita's theorem concerning generalized uniserial algebras, Proc. Japan Acad., 34 (1958), 404-406.

[6] K. Morita, Duality for modules and its applications to the theory of rings with minimum condition, Sci. Rep. Tokyo Kyoiku Daigaku, Sect. A, vol. 6, No. 150 (1958), 
83-142.

[ 7 ] T. Nakayama and T. Tsuzuku, On Frobenius extensions. I, Nagoya Math. J., 17 (1960), 89-110.

Department of Mathematics

Tokyo University of Education 